\title{
NF-kB-miR15a-bFGF/VEGFA axis contributes to the impaired angiogenic capacity of BM-MSCs in high fat diet-fed mice
}

\author{
ZHUOLI ZHU, XUEQI GAN and HAIYANG YU \\ State Key Laboratory of Oral Diseases, National Clinical Research Center for Oral Diseases, Department of Prosthodontics, \\ West China Hospital of Stomatology, Sichuan University, Chengdu 610041, P.R. China
}

Received October 10, 2016; Accepted June 13, 2017

DOI: $10.3892 / \mathrm{mmr} .2017 .7498$

\begin{abstract}
Potent paracrine properties, such as secretion of angiogenic cytokines and growth factors, have been considered essential for the function of mesenchymal stem cells (MSCs) in tissue regeneration and repair. The present study determined that bone marrow-derived mesenchymal stem cells from mice fed a high fat diet (HFD) had reduced pro-angiogenic capacity, as evident from the reduced expression of vascular endothelial growth factor A (VEGFA) and basic fibroblast growth factor (bFGF); therefore, a reduced number of branches was induced in the angiogenesis assay. Additionally, the present study determined that miR-15a, a putative microRNA targeting both VEGFA and bFGF, may simultaneously downregulate bFGF and VEGFA expression levels through the 3'-untranslated region. Inhibition of miR-15a using an antagonist restored the expression of VEGFA and bFGF under fatty acid treatment and thus the angiogenic capacity. Furthermore, the HFD and fatty acids treatments transcriptionally activated the expression of miR-15a via nuclear factor- $\kappa \mathrm{B}$. In conclusion, the findings of the present study revealed that inhibition of miR-15a may restore the therapeutic efficacy of mesenchymal stem cells in patients suffering from obesity.
\end{abstract}

\section{Introduction}

Bone marrow mesenchymal stem cells (BM-MSCs) are readily isolated multipotent cells that are easily expanded and efficiently induced to differentiate into osteoblast, chondrocytes or adipocytes (1-3). Bone marrow-derived MSCs, have been widely used as therapeutic agents in order to replace or repair injured tissues (1-3). In addition to their direct cell differentiation capacities, the therapeutic effects of MSCs are also attributed

Correspondence to: Dr Haiyang Yu, State Key Laboratory of Oral Diseases, National Clinical Research Center for Oral Diseases, Department of Prosthodontics, West China Hospital of Stomatology, Sichuan University, 14 3rd Section, Renmin Road, Chengdu 610041, P.R. China

E-mail: yhyang6812@foxmail.com

Key words: NF- $\mathrm{BB}$, angiogenesis, miR15a, VEGFA, bFGF, high fat diet to their paracrine capacity, particularly the ability to induce angiogenesis induction, under the tissue repair and regeneration conditions (4-6). Previous epidemiological and rodent studies associated obesity with deficient wound healing and insufficient angiogenesis (7-10), it remains to be determined how MSCs adaptation to pro-obesity diets alters the angiogenic potential of these cells to promote wound healing and tissue regeneration.

Angiogenesis, which is the formation of new blood vessels from existing capillaries, is essential for tissue repair and regeneration. Mechanistically, angiogenesis is a fine-tuned process which is regulated by various growth factors, including basic fibroblast growth factor (bFGF) and vascular endothelial growth factor A (VEGFA). bFGF and VEGFA are two of the most well-studied factors regulating angiogenesis $(11,12)$. Increased bFGF and VEGF levels may induce cell proliferation and migration in endothelial cells via activation the respective receptors on the cell surface $(13,14)$. Coordinated regulation of bFGF and VEGFA expression is required for the angiogenic capacity of MSCs.

Recently, microRNAs (miRNAs), which are 22-nucleotide long, non-coding small RNAs, were identified as the critical regulators of angiogenesis $(15,16)$. These angiogenesis-associated miRNAs act via either cleavage or post-transcriptional silencing of target mRNAs. For example, miR-503 may simultaneously target VEGFA and bFGF in the cancer (17). However, the miRNAs regulating angiogenesis factors remain to be fully elucidated, and there should be still some other angiogenic microRNAs to be identified.

The present study determined that BM-MSCs from the high fat diet (HFD) fed mice displayed reduced angiogenic capacity, together with reduced protein expression levels of VEGFA and bFGF. Meanwhile, the present study determined that HFD may transcriptionally activate miR-15a via nuclear factor $\mathrm{kB}(\mathrm{NF}-\kappa \mathrm{B})$ and induce miR-15a in turn simultaneously reduces the expression of VEGFA and bFGF. The current study established that the NF- $\mathrm{B}-\mathrm{miR} 15 \mathrm{a}-\mathrm{VEGFA} / \mathrm{bFGF}$ axis may contribute to the deficient angiogenic capacity in MSCs from obese patients and inhibition of miR-15a may restore the therapeutic efficacy of mesenchymal stem cells.

\section{Materials and methods}

Mice and HFD treatment. A total of 20 male $\mathrm{C} 57 \mathrm{Bl} / 6$ mice ( 20 g; supplied by Animal Center of Sichuan University, 
Chengdu, China) were housed in the Unit for Laboratory Animal Medicine in Sichuan University. Mice were allowed free access to food and water and were kept at $22^{\circ} \mathrm{C}$ on a 12-h light/dark cycle under specific pathogen-free conditions. Long-term HFD was achieved by feeding male mice a dietary chow consisting of $60 \% \mathrm{kcal}$ fat beginning at the age of 8-12 weeks and extending for a period of 6 months. Control mice were age-matched and fed standard chow ad libitum. The study was approved by the Ethics Committee of West China School of Stomatology, Sichuan University (Chengdu, China).

Cell isolation and culture. Cell isolation and culture were performed as previously described (18). Briefly, animals were sacrificed by intraperitoneal injection of a lethal dose of pentobarbital (West Tang Biotechnology, Inc., Shanghai, China; $100 \mathrm{mg} / \mathrm{kg}$ body weight). Tibias and femurs were harvested immediately following the animal euthanasia. Bone marrow cells were harvested by flushing cavities of tibia and femur bones with Dulbecco's modified Eagle's medium (DMEM)/F12 (Sigma-Aldrich Shanghai Trading Co., Ltd., Shanghai, China). Cells were centrifuged at $800 \mathrm{x}$ g for $5 \mathrm{~min}$ at $4^{\circ} \mathrm{C}$, re-suspended in complete medium [DMEM/F12 with $10 \%$ fetal bovine serum, Sigma-Aldrich; and penicillin/streptomycin, (Invitrogen, Thermo Fisher Scientific, Inc., Waltham, MA, USA)] and seeded at $30 \%$ confluence into a cell culture flask. Cells were cultured in at $37^{\circ} \mathrm{C}$ in $5 \% \mathrm{CO}_{2}$ for $72 \mathrm{~h}$ and non-adherent cells were removed. Cells were passaged with $0.25 \%$ trypsin/EDTA (Invitrogen, Thermo Fisher Scientific, Inc.) when reaching 90\% confluence. For the treatment of palmitic acid (PA, P0500; Sigma-Aldrich Shanghai Trading Co., Ltd.), PA was dissolved in $1 \%$ BSA (V900933; Sigma-Aldrich Shanghai Trading Co., Ltd.) and cells were treated with either BSA or $0.5 \mathrm{mmol} / 1$ of PA.

Transfection of miRNA mimics and antagonists. Transfection of miRNAs was performed as previously described (17). Briefly, miR-15a-5p mimics (sense: 5'-UAGCAGCACAUA AUGGUUUGUGdTdT-3'; antisense: 5'-CACAAACCAUUA UGUGCUGCUAdTdT-3'), inhibitors (antago-miR-15, with the single strand: 5'-CACAAACCAUUAUGUGCUGCU A-3') or the corresponding negative controls (NC) were synthesized in GenePharma Co. Ltd. (Shanghai, China). The cells were transfected with the aforementioned antagonists at final concentrations of $100 \mathrm{nM}$ with Lipofectamine 2000 (Invitrogen; Thermo Fisher Scientific, Inc.) as instructed by the manufacturer's protocol.

Reverse transcription-quantitative polymerase chain reaction (RT-qPCR). Total RNA was extracted with TRIzol (Invitrogen; Thermo Fisher Scientific, Inc.) according to manufacturer's protocol and the reverse transcription of miRNAs was performed using a miRNA RT reagent kit (Tiangen Biotech., Co., Ltd., Beijing, China). The RT reaction was run under $37^{\circ} \mathrm{C}$ for $30 \mathrm{~min}$, followed by inactivation at $85^{\circ} \mathrm{C}$. Expression of target mRNA VEGFA, bFGF and miR-15a levels were quantified using qPCR with the SYBR (TakaRa, Dalian, Liaoning, China) method. The cycling conditions were as follows: $95^{\circ} \mathrm{C}$ for $10 \mathrm{mins}, 95^{\circ} \mathrm{C}$ for $15 \mathrm{sec}, 60^{\circ} \mathrm{C}$ for $30 \mathrm{sec}$ and $72^{\circ} \mathrm{C}$ for $40 \mathrm{sec}(40 \mathrm{cycles})$ and $95^{\circ} \mathrm{C}$ for $60 \mathrm{sec}$, followed by dissociation curve analysis. Relative expression of target mRNA or miRNA were normalized to $\beta$-actin and U6 levels respectively. Primers used in the present study were as follows: VEGFA, forward (F) 5'-GCTTCCTACAGCACAGCAGA-3' and reverse (R) 5'-AATGCTTTCTCCGCTCTGAA-3'; bFGF, F 5'-GATGGA CGGCTGCTGGCTTCT-3' and R 5'-TGCCCAGTTCGTTTC AGTGCC-3'; $\beta$-actin F 5'-TTCTTTGCAGCTCCTTCGTT-3' and $\mathrm{R}$ 5'-ATGGAGGGGAATACAGCCC-3'. Primers for miR-15a and U6 were purchased from Tiangen Biotech., Co., Ltd. All the experiments were done at least three times. The $2^{-\Delta \Delta C q}$ method was used for quantification as described (19).

Bioinformatic analysis of miR-15a promoter. The promoter sequences of miR15a among different species using the web based ECR software were analyzed as instructed (20). Briefly, the gene name was inserted in the search box before entering the run key. The sequence conservation was shown by the bars with different colors indicating the promoter, exon, and intron respectively. The promoter region was selected. To further analyze the putative transcription factor binding sites in miR-15a, the 2,000 bp region of the Dleu2 promoter (in which miR-15a harbors) was further analyzed using JASPAR as instructed by the online prediction tool (21). All the putative p65 binding sites were thus selected for further biological study.

Chromatin immunoprecipitation (ChIP) analysis. ChIP was performed using an anti-p65 antibody (mAb 8242; 1:200; Cell Signaling Technology, Danvers, MA, USA) as previously described (22). Following ChIP, the DNA precipitated by the antibody was detected with qPCR. The PCR primer pairs for the miR-15a regulatory region were as follows: Primer set 1 : 5'-CCAGAGACGGAGGGAGGTAG-3' and 5'-CCGCACTCT GACCACTCGAC-3'; Primer set 2: 5'-AAGCCCCTCACA GTAGGTAG-3' and 5'-AAGTTAGCTACCAGATCACT-3', which amplify the regions around the two NF- $\mathrm{B}$ binding sites of deleted in lymphocytic leukemia 2 (Dleu2), whose intron harbors pre-miR-15a. The enrichment of p65 on miR15 upstream regulatory region was further calculated.

ELISA analysis of bFGF and VEGFA. The conditioned culture media from cells with the indicated treatments were collected for measuring secreted VEGFA and bFGF via ELISA. Briefly, the conditioned medium from MSCs ( $1 \mathrm{ml}$ from each well) was harvested and centrifuged at $\sim 6,000 \mathrm{x} \mathrm{g}$ for $15 \mathrm{~min}$ to exclude dead cells and debris. The supernatants were analyzed for the levels of secreted VEGF and bFGF protein using Quantikine VEGF (R\&D; MMV00; Bio-Techne China Co. Ltd., Shanghai, China) and bFGF ELISA kits (R\&D; MFB00; Bio-Techne China Co. Ltd.) according to the manufacturer's protocol.

Angiogenesis assay. Angiogenesis assay was performed as previously described (18). Briefly, 24-well plates were coated with Matrigel Matrix Grow Factor Reduced (BD Biosciences, Franklin Lakes, NJ, USA) (100 ml/well) and incubated at room temperature for $30 \mathrm{~min}$. Human umbilical vein endothelial cells were seeded on the plates and cultured with the conditioned medium from control, HFD fed mice-derived MSCs or cells with either control, indicated miRNA mimic or antagonist at a ratio of 50:50 with the fresh DMEM medium. Angiogenesis was analyzed under a light microscope (Nikon Corporation, 
Tokyo, Japan) at x20 magnification by calculating the branch number and length.

Luciferase reporter plasmids and dual luciferase reporter assay. The full length of the 3'-UTRs of VEGFA and part of the bFGF 3'UTR (covering the region of miR-15a recognition site) were synthesized and cloned into the pGL3-control vector (Promega Corporation, Madison, WI, USA) through the XbaI cloning site, where the 3'UTR fragment was inserted downstream of the firefly luciferase coding region. The sequencing (AUGCT sequencing company, Beijing, China) confirmed correct constructs were referred to as pMIR-bFGF-3'UTR and pMIR-VEGFA-3'UTR respectively. For the luciferase reporter assay, HEK293 cells (CRL-1573 ${ }^{\mathrm{TM}}$; American Type Culture Collection, Manassas, VA, USA) were cultured in 24-well plates in DMEM at $37^{\circ} \mathrm{C}$ and under $5 \% \mathrm{CO}_{2}$, and transfected with $200 \mathrm{ng}$ of either pMIR-bFGF-3'-UTR or pMIR-VEGFA-3'-UTR in combination with $100 \mathrm{nM}$ of miR-15a or non-target miRNA mimics, along with $10 \mathrm{ng}$ of pRL-TK vector containing Renilla luciferase (Promega Corporation) using Lipofectamine 2000 according to the manufacturer's the protocol. Cells were lysed with a passive lysis buffer (Promega Corporation) $48 \mathrm{~h}$ after the transfection and used for luciferase assays. Firefly and Renilla luciferase activities were quantified with Dual-Luciferase Reporter Assay System (Promega Corporation) and relative luciferase activity was calculated. Each experiment was performed in triplicate and repeated at least three times.

Subcellular fraction and western blotting. Mesenchymal stem cells were harvested for immunoblotting. For extraction of cytoplasmic and nuclear fraction, control or HFD fed mice-derived MSCs were sequentially incubated with cytoplasmic and nuclear extraction buffers (78833; NE-PER Nuclear and Cytoplasmic Extraction Reagents; Thermo Fisher Scientific, Inc.), and fractions containing cytosolic and nucleic proteins were collected at each step. Immunoblotting analysis was performed on cytosolic and nucleic fractions as previously described (18). Briefly, the protein concentration was quantified using BCA assay (Pierce BCA Protein Assay kit; 23225; Thermo Fisher Scientific, Inc.). Next, $20 \mu \mathrm{g}$ subcellular fraction or $60 \mu \mathrm{g}$ total cell extract of the cell lysates were separated by $12 \%$ SDS-PAGE and transferred to nitrocellulose membranes. The membrane was blocked with 5\% BSA (V900933; Sigma-Aldrich Shanghai Trading Co., Ltd.) before immunoblotting with specific antibodies against p65 (8242; 1:1,000; Cell Signaling Technology), Lamin B (ab16048; 1:1,000; Abcam), GAPDH (ab9485; 1:5,000; Abcam) and Tubulin (ab6046; 1:5,000; Abcam).

Statistical analysis. All experiments were repeated at least three times. Data were presented as mean \pm standard deviation. Statistical analysis was performed using the Student's t-test. $\mathrm{P}<0.05$ was considered to indicate a statistically significant difference.

\section{Results}

Decreased angiogenic capacity of BM-MSCs obtained from the HFD mice. To determine the differences of MSCs from healthy controls and HFD-fed mice, the present study quantified MSC abundance, proliferation and angiogenic capacity. No significant differences of MSC abundance and proliferation rate were identified between control and HFD mice (Fig. 1A). In addition, MSCs from both groups had a similar capacity to differentiation into osteoblasts (data not shown). Angiogenesis is considered to be essential for bone formation and also critical for MSC-mediated cell transplantation therapies. Therefore, the present study compared the angiogenic capacity of MSCs from control and HFD-fed mice. The conditioned medium of the passage 3 MSCs from the HFD mice, had a significantly reduced angiogenic ability (Fig. 1B and C). As VEGFA and bFGF are established potent angiogenic growth factors, their concentration in the culture medium was subsequently determined. The concentration of VEGFA and bFGF secreted by MSCs from the HFD-fed mice was significantly reduced (Fig. 1D). It is of note that there were no significant differences in the VEGFA and bFGF mRNA expression levels in the MSCs from control diet and HFD mice (Fig. 1E), suggesting that HFD affects the expression of VEGFA and bFGF post-transcriptionally.

miR-15a downregulates bFGF and VEGFA simultaneously. The reduced bFGF and VEGFA expression in HFD-fed mice-derived MSCs may be attributed to deregulated miRNAs. Analysis of the 3'-UTR of bFGF and VEGFA revealed that both may be regulated by miR-15a (Fig. 2A). The recognition site in the 3'-UTR is highly conserved among species (Fig. 2A), further suggesting the possible regulation of bFGF and VEGFA by miR-15a. Additionally, 2-fold increase in miR-15a expression was identified in HFD mice-derived BM-MSCs (Fig. 2B). Furthermore, miR-15a mimic transfection reduced the bFGF and VEGFA protein expression levels in MSCs compared with the chow diet derived MSCs (Fig. 2C), whereas the miR-15a antagonist restored their expression in MSCs from HFD-fed mice (data not shown). The present study determined that miR-15a reduced the luciferase activity of bFGF and VEGFA 3'-UTR reporter (Fig. 2D). The aforementioned data indicate that upregulation of miR-15a may be responsible for the reduction of bFGF and VEGFA expression in MSCs from HFD-fed mice.

Inhibition of $\mathrm{miR}-15$ a restores the angiogenic capacity of MSCs. Similar as the MSCs from the HFD-fed mice, 2-week exposure of palmitic acid also reduced the angiogenic factor expression of VEGFA and bFGF, consistent with the induction of miR-15a (Fig. 3A and B). By contrast, inhibition of miR-15a restored the VEGFA and bFGF expression levels (Fig. 3A and B). Inhibition of miR-15a restored the angiogenic capacity of MSCs from HFD-fed mice or exposed to palmitic acid (Fig. 3C-E). These data indicate that upregulation of miR-15a in MSCs from HFD-fed mice or palmitic acid-treated MSCs contribute significantly to the observed reduced angiogenic capacity.

$H F D$ induces the expression of miR-15a via 065 activation. Previous studies revealed that obesity may activate gene expression via transcriptional and post-transcriptional mechanisms (23-25). In order to determine the miR-15a aberrant expression in the MSCs from HFD-fed mice, the promoter 

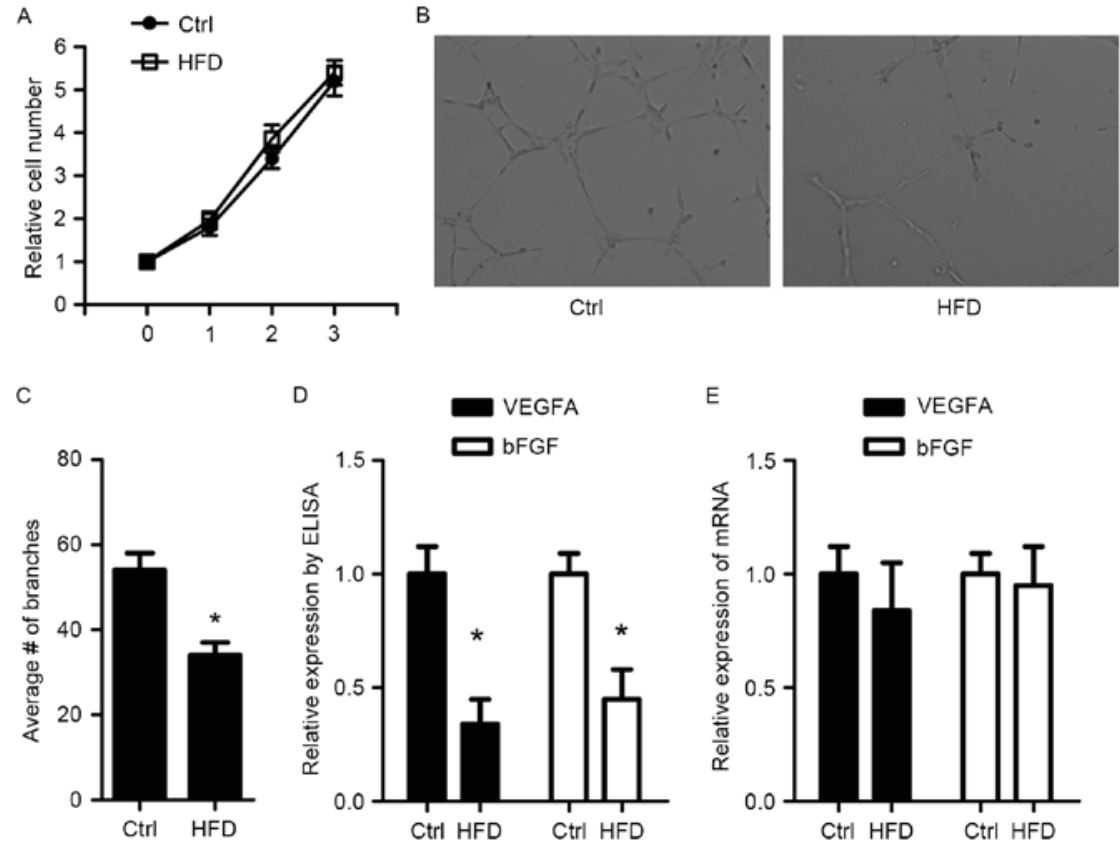

Figure 1. BM-MSCs from the HFD mice have reduced angiogenic capacity. (A) BM-MSCs from the high fat diet fed mice had no significant change in cell proliferation. BM-MSCs from the chow diet and HFD fed mice were isolated and passage 3 cells were grown in growth medium and cell proliferation rate was analyzed using Cell Counting Kit-8. (B) Reduced angiogenic capacity of BM-MSCs from the HFD-fed mice. The conditioned medium of BM-MSCs from chow diet or HFD fed mice was harvested for treatment of human umbilical vein endothelial cells in an angiogenesis assay. Representative images from three different experiments. (C) Quantitative data of the average branch numbers from the angiogenesis assay. $n=3,{ }^{*} \mathrm{P}<0.05$. (D) Decreased expression of VEGFA and bFGF in the conditioned medium of BM-MSCs from the HFD-fed mice. The conditioned medium of either BM-MSCs from chow diet or HFD-fed mice was harvested for ELISIA analysis of VEGFA and bFGF levels. $n=3$, and ${ }^{*} \mathrm{P}<0.05$. (E) No significant difference was identified between VEGFA and $b F G F$ at mRNA levels of BM-MSCs from the HFD-fed mice and the Ctrl mice. BM-MSCs from chow diet or HFD-fed mice were harvested for RNA purification, and expression of VEGFA and bFGF at mRNA level was analyzed using reverse transcription-quantitative polymerase chain reaction. $\mathrm{n}=3$, ${ }^{*} \mathrm{P}<0.05$. HFD, high fat diet; Ctrl, control; VEGFA, vascular endothelial growth factor A; bFGF, basic fibroblast growth factor; BM-MSCs, bone marrow mesenchymal stem cells .

A

\begin{tabular}{|c|c|}
\hline & \\
\hline Mouse VEGFA & UUUUCUUAUAUCUGCUGCUA \\
\hline Human VEGFA & UUUUAUUUUUCUUGCUGCUA \\
\hline Rat VEGFA & UUUUCUUAUAUUUGCUGCUA \\
\hline miR-15a & 3'-GUGUUUGGUAAUACACGACGAU-5' \\
\hline Mouse bFGF & CUUCACGGAACUCAGCUGCUA \\
\hline Human bFGF & AGAAUCUUACAGAUGCUGCUA \\
\hline Rat bFGF & AGGAUCUUACAGAUGCUGCUA \\
\hline
\end{tabular}

B

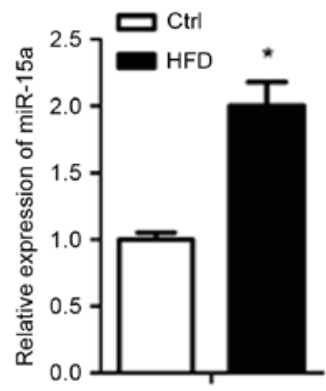

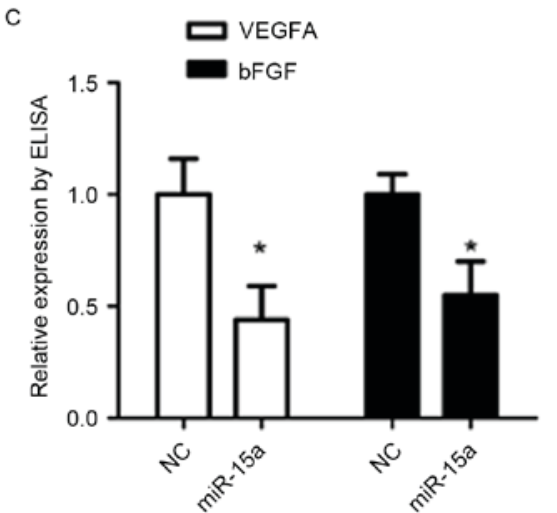

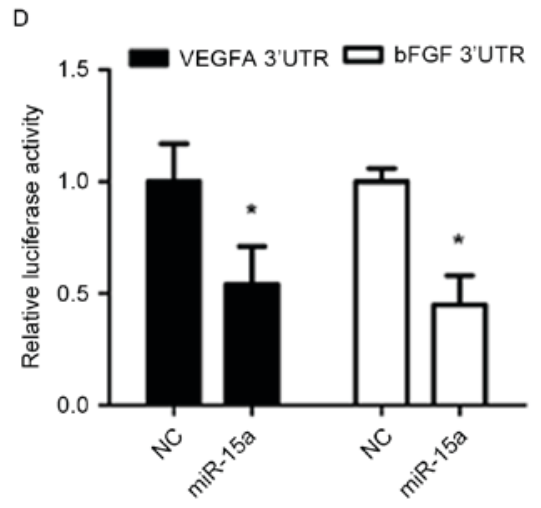

Figure 2. miR-15a simultaneously downregulates bFGF and VEGFA. (A) VEGFA (upper) and bFGF (lower) 3'-UTR contain the conservative binding site for miR-15a. (B) Increased expression of miR-15a was observed in the BM-MSCs from high fat diet-fed mice. (C) miR-15a reduces the expression of bFGF and VEGFA in BM-MSCs. BM-MSCs from chow diet mice were treated with miR-15a and expression of bFGF and VEGFA in the medium was analyzed by ELISA. $\mathrm{n}=3$, "P $<0.05$. (D) miR-15a reduces the luciferase activity of bFGF and VEGFA 3'-UTR reporter. bFGF and VEGFA 3'-UTR reporter plasmids were co-transfected with control and miR-15a as indicated, with pRL-TK as internal control. $\mathrm{n}=5$, $\mathrm{P}<0.05$. miR-15a, microRNA-15a; VEGFA, vascular endothelial growth factor A; bFGF, basic fibroblast growth factor; 3'-UTR, 3'-untranslated region BM-MSCs, bone marrow mesenchymal stem cells; NC, negative control. 
A

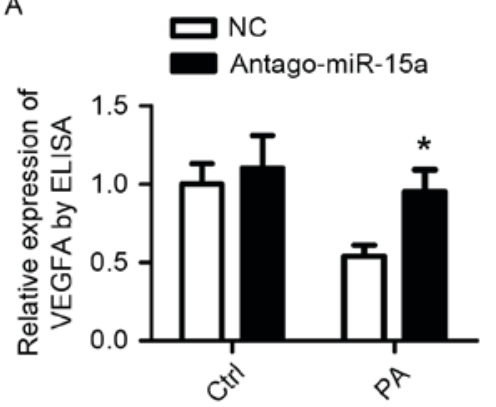

B

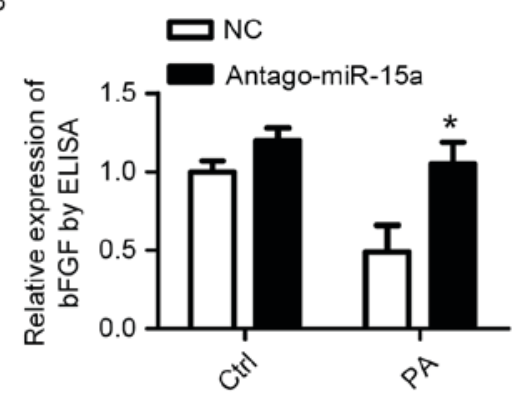

C

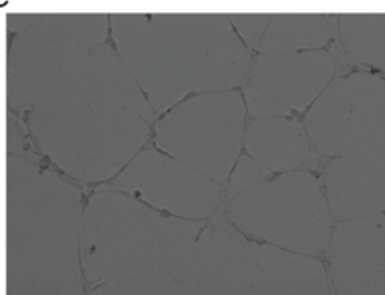

Ctrl+NC

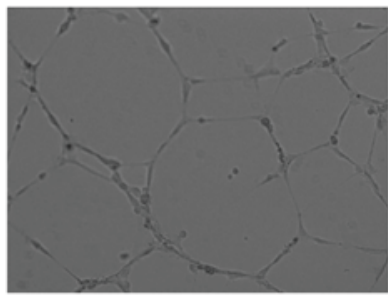

Ctrl+antago-miR-15a

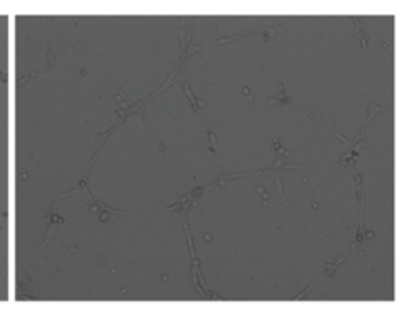

$\mathrm{PA}+\mathrm{NC}$

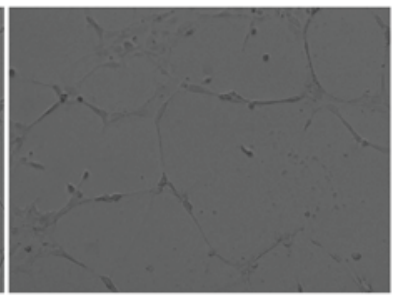

PAtantago-miR-15a
D

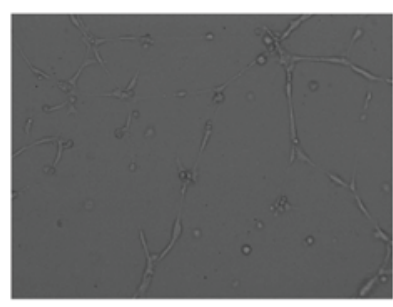

$\mathrm{HFD+NC}$

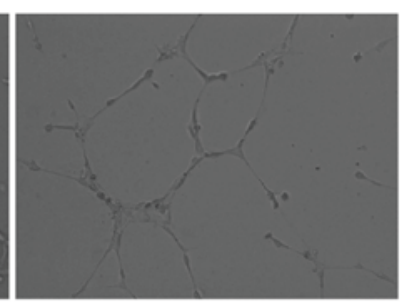

HFD+antago-miR-15a

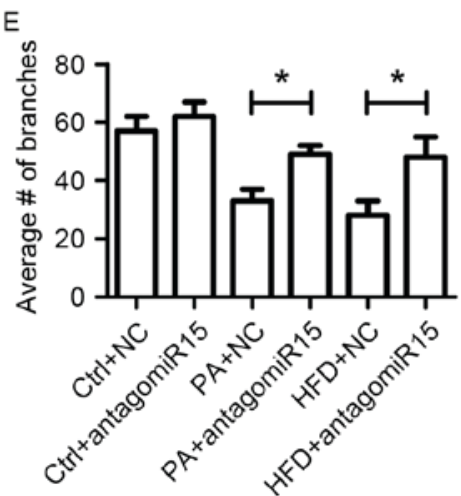

Figure 3. Inhibition of miR-15a restores the angiogenic capacity of MSCs from HFD fed mice. (A) Inhibition of miR-15a restores the expression of VEGFA and (B) bFGF in MSCs treated with PA, n=3. (C) Inhibition of miR-15a restores the angiogenic ability of MSCs treated with PA. n=3. (D) Inhibition of miR-15a restores the angiogenic capacity of BM-MSCs from HFD fed mice. $n=3$. (E) Quantitative data of the average branch numbers from the angiogenesis assays. $\mathrm{n}=3$, $\mathrm{P}<0.05$. miR-15a, microRNA-15a; VEGFA, vascular endothelial growth factor A; bFGF, basic fibroblast growth factor; HFD, high fat diet; PA, palmitic acid; BM-MSCs, bone marrow mesenchymal stem cells; Ctrl, control; NC, negative control.

sequences of miR15a among different species using the web based ECR software were analyzed (20). miR-15a is imbedded in the intron of Dleu2 gene (Fig. 4A), which has conserved upstream regulatory regions. The locus of miR-15a suggests that miR15-a may share a regulatory mechanism with Dleu2. Therefore, the 2,000 bp region of the Dleu2 promoter was further analyzed using JASPAR (21). There were two highly conserved RELA binding sites (Fig. 4A), suggesting that $\mathrm{NF}-\kappa \mathrm{B}$ may be involved in miR-15a aberration. Accordingly, the present study determined that there was higher nuclear translocation of p65 in the HFD mice-derived MSCs (Fig. 4B). ChIP analysis using the anti-p65 antibody revealed enrichment of p65 around the putative two RELA binding sites in the miR-15a (Dleu2) regulatory region in MSCs from HFD-fed mice (Fig. 4C). Furthermore, exposure to the NF- $\kappa$ B inhibitor, BAY 11-708, limited the induction of miR-15a in the MSCs from HFD-fed mice and had a marginal effect on the MSCs from mice fed with normal chow (Fig. 4D).

\section{Discussion}

The present study identified that HFD reduced the angiogenic capacity of MSCs. Additionally, it was revealed that HFD transcriptionally activates miR-15a, which in turn acts as a 


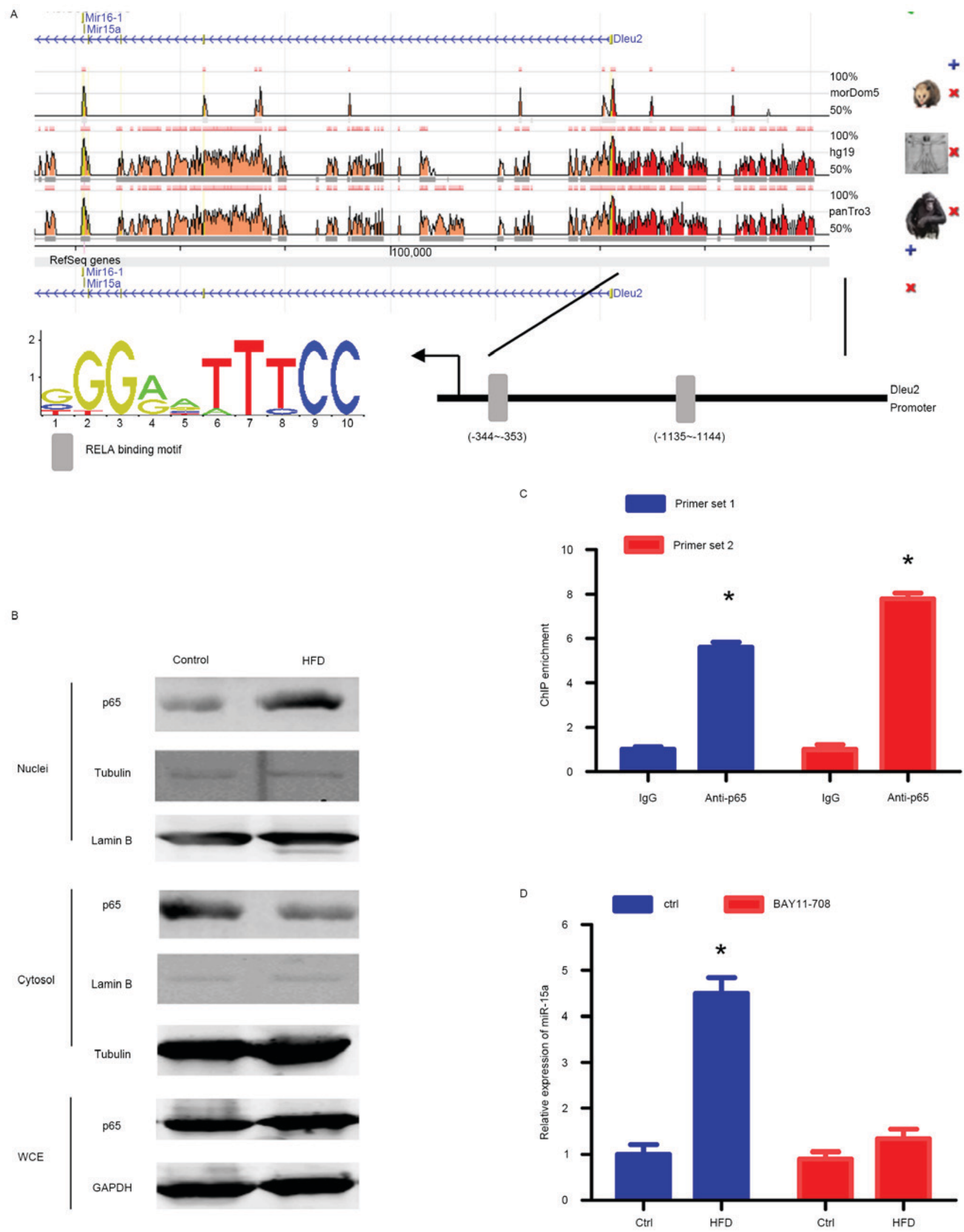

Figure 4. HFD upregulates miR-15a expression via p65. (A) miR-15a is located in the intron region of non-coding Dleu2 and the regulatory region is conserved across species, as presented by the ECR online tool (20). The upstream 2,000 bp promoter region of mouse Dleu2 was analyzed for the putative nuclear factor- $\mathrm{kB}$ binding site by JASPAR (21) was noted. (B) HFD treatment induced the nuclear translocation of p65 in BM-MSCs. Nuclear translocation of p65 was analyzed by western blotting. Lamin B, GAPDH and tubulin were used as the loading control for nuclei, cytosol and WCE, respectively. Data were representative of 3 different experiments. (C) Anti-p65 chromatin immunoprecipitation analysis revealed enrichment of p65 in the two putative RELA binding sites of miR-15 regulatory region in BM-MSCs from HFD-fed mice. Primer set 1 covered the -344 -353 region and Primer set 2 covered the $-1,135 \sim-1,144$ region. $n=3$, ${ }^{*} \mathrm{P}<0.05$. (D) BM-MSCs from chow diet and HFD-fed mice were treated with vehicle or BAY 11-708 from the beginning of isolation and expression of miR-15a and were analyzed using quantitative polymerase chain reaction in passage 1. $\mathrm{n}=3$, "P<0.05. HFD, high fat diet; BM-MSCs, bone marrow mesenchymal stem cells; Ctrl, control; Dleu2, deleted in lymphocytic leukemia 2; WCE, whole cell extract; RELA, RELA proto-oncogene, NF-kB subunit; BAY 11-708, nuclear factor-kB inhibitor. 
novel potent angiogenic inhibitor in MSCs by simultaneously downregulating bFGF and VEGFA. It has been previously established that metabolism may transcriptionally and epigenetically alter gene expression, whereas the specific changes in different cell types and genes remain to be elucidated. The present study identified that HFD induced activation of $\mathrm{NF}-\kappa \mathrm{B}$, which in turn binds to the RELA elements on the promoter/regulatory region of miR-15a and contributes to the increase of miR-15a in MSCs from HFD mice. The present study determined that inhibition of miR-15a may restore the therapeutic efficacy of mesenchymal stem cells by upregulation of its angiogenic capacity. The current study established that HFD-miR15-VEGFA/bFGF axis may contribute to the deficient angiogenic capacity in MSCs from obese patients. It is important to note that we only observed tube formation and VEGF and FGF expression in the present study. Future work using aortic ring assay and additional angiogenic models may confirm the present conclusion and provide novel information on the effect of the HFD-miR15-VEGFA/bFGF axis on angiogenesis process. Additionally, the underlying mechanism of how a HFD and increased NF- $\kappa \mathrm{B}$ expression induces miR-15a remains to be elucidated. It is has been previously established that activated $\mathrm{NF}-\kappa \mathrm{B}$ may recruit histone acetyltransferase and thus induce the histone acetylation in the target gene (26). Therefore, future studies may investigate the histone acetylation status of the miR-15a (Dleu2) regulatory region. It is of note that the miR-15 miRNA precursor family includes the miR-15a and miR-15b, miR-16-1, miR-16-2, miR-195 and miR-497 sequences $(27,28)$. These six highly conserved miRNAs are clustered on three separate chromosomes, raising the possibility that other members of the miR-15 family may be also involved in the reduced VEGFA and bFGF expression levels observed in HFD mice. In addition, the miR-15a antagonist partially restored the expression of bFGF and VEGFA, suggesting that additional mechanisms may exist for the reduced expression of bFGF and VEGFA in MSCs from HFD mice.

miR-15 has been previously identified as a tumor suppressor $(27,28)$, the present study further elucidated the tumor suppressor role of miR-15a, particularly when angiogenesis is considered as the most potent promoter of cancer progress. Obesity is linked to colon cancer; therefore, it is interesting to determine whether the same mechanism occurs during colon cancer initiation and progression. Additionally, previous studies have linked obesity to osteoporosis (29-32) and insufficient wound repair (7-10); therefore, it is possible that the HFD-miR15-VEGFA/bFGF axis, as aforementioned, may contribute to obesity-associated osteoporosis and other defects.

In conclusion, the present study revealed that the transcriptional activation of miR-15a may partially contribute to the deficient angiogenic capacity of MSCs in HFD-fed mice, raising the possibility that inhibition of miR-15 may restore the therapeutic efficacy of MSCs from obese patients.

\section{Acknowledgements}

The present study was supported by a grant from the Ministry of Education of the People's Republic of China (grant no. 20120181120007).

\section{References}

1. Cerqueira MT, Pirraco RP and Marques AP: Stem cells in skin wound healing: Are we there yet? Adv Wound Care (New Rochelle) 5: 164-175, 2016.

2. Martin I, De Boer J and Sensebe L; MSC Committee of the International Society for Cellular Therapy: A relativity concept in mesenchymal stromal cell manufacturing. Cytotherapy 18: 613-620, 2016.

3. Saeed H, Ahsan M, Saleem Z, Iqtedar M, Islam M, Danish Z and Khan AM: Mesenchymal stem cells (MSCs) as skeletal therapeutics-an update. J Biomed Sci 23: 41, 2016.

4. King A, Balaji S, Keswani SG and Crombleholme TM: The role of stem cells in wound angiogenesis. Adv Wound Care (New Rochelle) 3: 614-625, 2014.

5. Pountos I, Panteli M, Panagiotopoulos E, Jones E and Giannoudis PV: Can we enhance fracture vascularity: What is the evidence? Injury 45 (Suppl 2): S49-S57, 2014.

6. Tamama K and Kerpedjieva SS: Acceleration of wound healing by multiple growth factors and cytokines secreted from multipotential stromal cells/mesenchymal stem cells. Adv Wound Care (New Rochelle) 1: 177-182, 2012.

7. David MA, Jones KH, Inzana JA, Zuscik MJ, Awad HA and Mooney RA: Tendon repair is compromised in a high fat diet-induced mouse model of obesity and type 2 diabetes. PLoS One 9: e91234, 2014.

8. Kato Y, Iwata T, Morikawa S, Yamato M, Okano T and Uchigata Y: Allogeneic transplantation of an adipose-derived stem cell sheet combined with artificial skin accelerates wound healing in a rat wound model of type 2 diabetes and obesity. Diabetes 64: 2723-2734, 2015.

9. White TJ, Santos MC and Thompson JS: Factors affecting wound complications in repair of ventral hernias. Am Surg 64: 276-280, 1998.

10. Xing L, Culbertson EJ, Wen Y, Robson MC and Franz MG: Impaired laparotomy wound healing in obese rats. Obes Surg 21: 1937-1946, 2011.

11. Folkman J: Angiogenesis and angiogenesis inhibition: An overview. Exs 79: 1-8, 1997.

12. Pepper MS, Mandriota SJ, Vassalli JD, Orci L and Montesano R: Angiogenesis-regulating cytokines: Activities and interactions. Curr Top Microbiol Immunol 213: 31-67, 1996.

13. Dworacka M, Krzyżagórska E, Wesolowska A, Borowska M, Iskakova S and Dworacki G: Statins in low doses reduce VEGF and bFGF serum levels in patients with type 2 diabetes mellitus. Pharmacology 93: 32-38, 2014.

14. Wilcke I, Lohmeyer JA, Liu S, Condurache A, Krüger S Mailänder P and Machens HG: VEGF (165) and bFGF protein-based therapy in a slow release system to improve angiogenesis in a bioartificial dermal substitute in vitro and in vivo. Langenbecks Arch Surg 392: 305-314, 2007.

15. Wang S, Aurora AB, Johnson BA, Qi X, McAnally J, Hill JA, Richardson JA, Bassel-Duby R and Olson EN: The endothelial-specific microRNA miR-126 governs vascular integrity and angiogenesis. Dev Cell 15: 261-271, 2008.

16. Bonauer A, Carmona G, Iwasaki M, Mione M, Koyanagi M, Fischer A, Burchfield J, Fox H, Doebele C, Ohtani K, et al: MicroRNA-92a controls angiogenesis and functional recovery of ischemic tissues in mice. Science 324: 1710-1713, 2009.

17. Zhou B,Ma R,Si W,Li S, Xu Y, Tu X and Wang Q: MicroRNA-503 targets FGF2 and VEGFA and inhibits tumor angiogenesis and growth. Cancer Lett 333: 159-169, 2013.

18. Zhu Z, Gan X, Fan H and Yu H: Mechanical stretch endows mesenchymal stem cells stronger angiogenic and anti-apoptotic capacities via NFKB activation. Biochem Biophys Res Commun 468: 601-605, 2015.

19. Livak KJ and Schmittgen TD: Analysis of relative gene expression data using real-time quantitative PCR and the 2(-Delta Delta C(T)) method. Methods 25: 402-408, 2001.

20. Ovcharenko I, Nobrega MA, Loots GG and Stubbs L: ECR Browser: A tool for visualizing and accessing data from comparisons of multiple vertebrate genomes. Nucleic Acids Res 32: W280-W286, 2004.

21. Mathelier A, Zhao X, Zhang AW, Parcy F, Worsley-Hunt R, Arenillas DJ, Buchman S, Chen CY, Chou A, Ienasescu H, et al: JASPAR 2014: An extensively expanded and updated open-access database of transcription factor binding profiles. Nucleic Acids Res 42 (Database Issue): D142-D147, 2014.

22. Yang G, Lu X, Wang L, Bian Y, Fu H, Wei M, Pu J, Jin L, Yao L and Lu Z: E2F1 and RNA binding protein QKI comprise a negative feedback in the cell cycle regulation. Cell Cycle 10: 2703-2713,2011. 
23. Burgio E, Lopomo A and Migliore L: Obesity and diabetes: From genetics to epigenetics. Mol Biol Rep 42: 799-818, 2015.

24. Cordero P, Li J and Oben JA: Epigenetics of obesity: Beyond the genome sequence. Curr Opin Clin Nutr Metab Care 18: 361-366, 2015.

25. Lee BC and Lee J: Cellular and molecular players in adipose tissue inflammation in the development of obesity-induced insulin resistance. Biochim Biophys Acta 1842: 446-462, 2014.

26. Li D, Huang ZZ, Ling YZ, Wei JY, Cui Y, Zhang XZ, Zhu HQ and Xin WJ: Up-regulation of CX3CL1 viaNuclearFactor- $\kappa B$-dependent histone acetylation is involved in paclitaxel-induced peripheral neuropathy. Anesthesiology 122: 1142-1151, 2015

27. Bottoni A, Piccin D, Tagliati F, Luchin A, Zatelli MC and degli Uberti EC: miR-15a and miR-16-1 down-regulation in pituitary adenomas. J Cell Physiol 204: 280-285, 2005.

28. Cimmino A, Calin GA, Fabbri M, Iorio MV, Ferracin M, Shimizu M, Wojcik SE, Aqeilan RI, Zupo S, Dono M, et al: miR-15 and miR-16 induce apoptosis by targeting BCL2. Proc Natl Acad Sci USA 102: 13944-13949, 2005.
29. Zhao LJ, Jiang H, Papasian CJ, Maulik D, Drees B, Hamilton J and Deng HW: Correlation of obesity and osteoporosis: effect of fat mass on the determination of osteoporosis. J Bone Miner Res 23: 17-29, 2008.

30. Colaianni G, Brunetti G, Faienza MF, Colucci S and Grano M: Osteoporosis and obesity: Role of Wnt pathway in human and murine models. World J Orthop 5: 242-246, 2014.

31. Ilich JZ, Kelly OJ, Kim Y and Spicer MT: Low-grade chronic inflammation perpetuated by modern diet as a promoter of obesity and osteoporosis. Arh Hig Rada Toksikol 65: 139-148, 2014.

32. Sharma S, Tandon VR, Mahajan S, Mahajan V and Mahajan A: Obesity: Friend or foe for osteoporosis. J Midlife Health 5: 6-9, 2014. 